\title{
BMJ Open Is prevalence of e-cigarette and nicotine replacement therapy use among smokers associated with average cigarette consumption in England? A time-series analysis
}

To cite: Beard E, Brown J, Michie S, et al. Is prevalence of e-cigarette and nicotine replacement therapy use among smokers associated with average cigarette consumption in England? A time-series analysis. BMJ Open 2018;8:e016046. doi:10.1136/ bmjopen-2017-016046

- Prepublication history and additional material for this paper are available online. To view these files, please visit the journal online (http://dx.doi org/10.1136/bmjopen-2017016046).

Received 23 January 2017 Revised 21 June 2017 Accepted 11 September 2017

Check for updates

${ }^{1}$ Cancer Research UK Health Behaviour Research Centre, University College London, London, UK

${ }^{2}$ Research Department of Educational, Clinical and Health Psychology, University College London, London, UK

Correspondence to

Dr Emma Beard;

e.beard@ucl.ac.uk

\section{ABSTRACT}

Objectives Many smokers use e-cigarettes and licensed nicotine replacement therapy (NRT), often in an attempt to reduce their cigarette consumption. We estimated how far changes in prevalence of e-cigarette and NRT use while smoking were accompanied by changes in cigarette consumption at the population level.

Design Repeated representative cross-sectional population surveys of adults aged 16+ years in England. Methods We used Autoregressive Integrated Moving Average with Exogeneous Input (ARIMAX) modelling of monthly data between 2006 and 2016 from the Smoking Toolkit Study. Prevalence of e-cigarette use and NRT use in current smokers, and specifically for smoking reduction and temporary abstinence, were input variables. Mean daily cigarette consumption was the dependent variable. Analyses involved adjustment for mass media expenditure and tobacco-control policies.

Results No statistically significant associations were found between changes in use of e-cigarettes $(\beta-0.012$, $95 \% \mathrm{Cl}-0.026$ to 0.002$)$ or NRT $(\beta 0.015,95 \% \mathrm{Cl}-0.026$ to 0.055 ) while smoking and daily cigarette consumption. Neither did we find clear evidence for an association between e-cigarette use $(\beta-0.010,95 \% \mathrm{Cl}-0.025$ to 0.005 and $\beta 0.011,95 \%-0.027$ to 0.004 ) or NRT use ( $\beta$ $0.006,95 \%-0.030$ to 0.043 and $\beta 0.022,95 \%-0.020$ to 0.063 ) specifically for smoking reduction and temporary abstinence, respectively, and changes in daily cigarette consumption

Conclusion If use of e-cigarettes and licensed NRT while smoking acted to reduce cigarette consumption in England between 2006 and 2016, the effect was likely very small at a population level.

\section{INTRODUCTION}

Randomised controlled trials have shown that use of non-tobacco nicotine-containing products (eg, nicotine replacement therapy; NRT) are efficacious for harm-reduction attempts. ${ }^{1}$ Harm reduction is defined as any attempt to reduce the harm from smoking without an intention to quit completely, such as, the use of NRT for smoking reduction (ie, during
Strengths and limitations of this study

- This is the first time series study to assess the population-level impact of the use of nicotine replacement therapy and e-cigarettes for harm reduction on cigarette consumption.

- This study uses a large representative sample of the population in England and considers both smoking reduction and temporary abstinence.

- A wide range of confounders are adjusted for including population-level interventions.

- In countries with weaker tobacco control, or stricter regulation of using products for harm reduction, different effects may be observed.

- Data are observational and so strong conclusions regarding cause and effect cannot be made.

attempts to cut down) or during periods of temporary abstinence (ie, during periods of time when one is unable to smoke). ${ }^{1}$ Outside of the clinical setting where little behavioural support is provided, the use of NRT during attempts to cut down smoking appears to increase smoker's propensity to quit, but does not result in significantly large reductions in cigarette consumption. ${ }^{2-4}$ Explanations for this include the lack of behavioural support and possible poor compliance with the medical regimen. ${ }^{56}$

In recent years, there has been an increase in the overall use of nicotine-containing products for harm reduction, with a growth in e-cigarettes more than offsetting a decline in the use of NRT. ${ }^{7-9}$ Previous studies suggest that e-cigarettes which contain nicotine reduce cravings more effectively than NRT, ${ }^{71011}$ have better adherence rates ${ }^{712}$ and deliver clinically significant levels of nicotine into the blood, at least for some smokers. ${ }^{1011} 13$ Thus, although further studies are needed it is possible that e-cigarettes may be a more 
effective aid for smoking reduction than licensed nicotine products. ${ }^{14}{ }^{15}$ However, it also remains possible that e-cigarettes will not result in clinically significant reductions in cigarette intake at a population level.

The aim of this study was to assess the association between changes in prevalence of e-cigarettes and NRT with changes in mean cigarette consumption per day using a time-series approach. Time-series analysis allows us to take into account underlying trends, the effect of other tobacco-control interventions, autocorrelation (whereby data collected at points closer in time tend to be more similar), and to consider possible lag effects of the independent variable on the dependent variable. ${ }^{16}$ Where associations are found, they cannot unequivocally establish a causal association but can be indicative, as has been the case with estimating the effect of price of cigarettes on population consumption, ${ }^{17}$ mass-media expenditure on use of specialist stop-smoking services ${ }^{18}$ and introduction of varenicline to the market on prevalence of use of smoking cessation medication. ${ }^{19}$ Where associations are not found, or they go in a direction opposite to that expected, this can also be informative.

Specifically, this paper assesses the association between mean cigarette consumption per day and:

1. Current e-cigarette use among smokers for any purpose, current use specifically for smoking reduction and current use specifically for temporary abstinence.

2. Current NRT use among smokers for any purpose, current use specifically for smoking reduction and current use specifically for temporary abstinence.

Sensitivity analyses will examine the effect of focusing only on daily e-cigarette and NRT use, given previous associations between extent of non-tobacco nicotine-containing product use and the effectiveness of harm-reduction attempts. ${ }^{6}$

\section{METHODS}

\section{Design}

We used Autoregressive Integrated Moving Average with Exogeneous Input (ARIMAX) modelling of monthly data between 2006 and 2016 primarily from the Smoking Toolkit Study. The smoking toolkit study (STS) is a monthly survey of a representative sample of the population in England aged 16+ years. ${ }^{20}$ This has been collecting data on smoking patterns among smokers and recent ex-smokers since November 2006. Questions on the use of e-cigarettes among all smokers were introduced in May 2011 and as aids to a quit attempt among smokers attempting to stop in July 2009. The STS involves monthly household surveys using a random location sampling design, with initial random selection of grouped output areas (containing 300 households), stratified by ACORN (sociodemographic) characteristics (https://acorn.caci.co.uk/) and region. Interviewers then choose which houses within these areas are most likely to fulfil quotas based on the probability of individuals being at home in different regions and conduct face-to-face computer-assisted interviews with one member per household. Participants from the STS appear to be representative of the population in England, having similar sociodemographic composition as other large national surveys, such as the Health Survey for England. ${ }^{20}$

\section{Measures}

Explanatory variables

Daily and non-daily smokers were asked the following questions:

1. Which, if any, of the following are you currently using to help you cut down the amount you smoke?

2. Do you regularly use any of the following in situations when you are not allowed to smoke?

3. Can I check, are you using any of the following either to help you stop smoking, to help you cut down or for any other reason at all?

All three questions had the following response options: nicotine gum, nicotine replacement lozenges tablets, nicotine replacement inhaler, nicotine replacement nasal spray, nicotine patch, electronic cigarette, nicotine mouth spray, other, none.

Current e-cigarette use was derived by an 'electronic cigarette' response to any of the three questions; e-cigarette use for smoking reduction by a response to the first question; and e-cigarette use for temporary abstinence by a response to the second question.

Current NRT use was derived by an NRT product response ('nicotine gum, nicotine replacement lozenges $\backslash$ tablets, nicotine replacement inhaler, nicotine replacement nasal spray, nicotine patch or nicotine mouth spray') to any of the three questions; NRT use for smoking reduction by an NRT product response to the first question; and NRT use for temporary abstinence by an NRT product response to the second question.

Data were not recorded on NRT use for temporary abstinence between November 2006 and January 2007 and was imputed using prevalence data from February 2007.

Data were only available on the prevalence of use of electronic cigarettes among smokers from April 2011 although use specifically during a recent quit attempt were available from July 2009. Thus, prevalence of electronic cigarette use among smokers between July 2009 and April 2011 was estimated from data on use during a quit attempt; use of electronic cigarettes among smokers between November 2006 and June 2009 was assumed to be $0.1 \%$ of smokers based on other surveys which found their use to be very rare before $2009 .^{21} 22$

Daily NRT and e-cigarette users were classified as those who reported that they used the product(s) at least once per day in response to the question: How many times per day on average do you use your nicotine replacement product or products? This question was introduced in July 2010. Prior to this time, prevalence of daily NRT use was assumed to be $60 \%$ of all users, ${ }^{6}$ while e-cigarette prevalence was computed as above using prevalence during a quit attempt or $0.1 \%$. 


\section{Outcome variables}

Smokers taking part in the STS were also asked how many cigarettes they smoke on average per day. Non-daily smokers were asked how many cigarettes they smoked per week which was then converted to a daily figure.

\section{Co-variables}

In England, tobacco mass media campaigns have been run as part of a national tobacco-control programme. Spending was almost completely suspended in 2010 and then reintroduced in 2011 at a much lower level. Previous studies have shown that such cuts were associated with a decreased use of smoking cessation support. ${ }^{18}{ }^{23}$ Thus, advertising expenditure will be adjusted for using data obtained from Public Health England. Data on mass media expenditure was available monthly from May 2008, and yearly prior to this period, and so a monthly average was assumed. For a number of months, spending was effectively zero and was imputed as 0.1 to allow the analysis to run.

A number of tobacco-control policies were adjusted for. These included the move in commissioning of stopsmoking services to local authorities in April 2013, ${ }^{24}$ introduction of a smoking ban in July $2007,{ }^{25}$ licensing of NRT for harm reduction in December 2009, ${ }^{26}$ the publication of National Institute for Health and Care Excellence guidance on harm reduction in June $2013^{27}$ and change in the minimum age of sale of cigarettes in October 2007. ${ }^{28}$ Price of cigarettes is correlated 0.99 with time and will thereby be taken into account by use of differencing (ie, using the differences between consecutive observation rather than observations themselves) to make the series stationary.

\section{Analysis}

The analysis plan was registered on the Open Science Framework prior to data analysis (https://osf.io/6swk3/). All data were analysed in R V.3.2.4 ${ }^{29}$ using ARIMAX modelling. ${ }^{163031}$ Data were weighted prior to the analyse to match the population in England using a rim (marginal) weighting technique. This involves an iterative sequence of weighting adjustments whereby separate nationally representative target profiles are set (for gender, working status, children in the household, age, social grade and region). This process is then repeated until all variables match the specified targets. ${ }^{20}$

Two waves of data were collected in March 2007 and March 2013. These waves were averaged. No data were collected in December 2008. Mean cigarette consumption, NRT use and e-cigarette use during this period were calculated as an average of the month before and the month after. For a few months (May 2012, July 2012, September 2012, November 2012, January 2013, March 2013), data on electronic cigarettes and NRT use among smokers were not recorded. For these months, the average of the previous and next month was imputed.

The Granger causality test suggested that there was some evidence for the violation of the assumption of weak exogeneity (ie, $\mathrm{Y}$ can depend on the lagged values of $\mathrm{X}$ but the reverse must not be true) between the input and the output series. However, caution has been advised when using this and similar tests on data across a long time series, ${ }^{32}{ }^{33}$ and there was no theoretical reason we could identify for a bidirectional relationship between e-cigarette use and cigarette consumption. It was assumed that the association was spurious and likely removed following adjustment for other covariates.

Both unadjusted and fully adjusted models are reported which regressed onto mean cigarette consumption per day: (1) use of e-cigarettes among current smokers; (2) use of e-cigarettes for smoking reduction; (3) use of e-cigarettes for temporary abstinence; (4) use of NRT for harm reduction; (5) use of NRT for temporary abstinence and (6) use of NRT for smoking reduction. Sensitivity analyses were conducted which constrained the analysis to only those reporting daily e-cigarette and NRT use. We followed a standard ARIMAX modelling approach. ${ }^{16} 34$ The series were first log-transformed to stabilise the variance, and if required, first differenced and seasonally differenced. The autocorrelation and partial autocorrelation functions were then examined in order to determine the seasonal and non-seasonal moving average (MA) and autoregressive terms (AR). For example, AR(1) means that the value of a series at one point in time is the sum of a fraction of the value of the series at the immediately preceding point in time and an error component; while MA(1) means that the value of a series at one point in time is a function of a fraction of the error component of the series at the immediately preceding point in time and an error component at the current point in time. To identify the most appropriate transfer function (ie, lag) for the continuous explanatory variables, the sample cross-correlation function was checked for each ARIMAX model. Coefficients can be interpreted as estimates of the percentage change in cigarette consumption for every (a) percentage increase in use of e-cigarettes and NRT, (b) percentage increase in mass media expenditure and (c) implementation of tobacco-control policies.

Bayes factors (BFs) were derived for non-significant findings using an online calculator ${ }^{35}$ to disentangle whether there is evidence for the null hypothesis of no effect $(\mathrm{BF}<1 / 3 \mathrm{rd})$ or the data are insensitive (BF between $1 / 3$ rd and 3). A half-normal distribution was assumed with a percentage change in the outcomes of interest for every percentage increase in the input series of $0.009 \%$ based on the effect detectable with $80 \%$ power (see sample size). Sensitivity analyses were conducted using a much larger percentage change of 0.1 . This was based on a meta-analysis assessing the efficacy of non-tobacco nicotine replacement products for harm reduction which reported that $21.8 \%$ of the experimental group had reduced consumption by more than $50 \%$ at final follow-up compared with $16.5 \%$ receiving placebo. ${ }^{1}$ We therefore assumed that a $5 \%$ change in prevalence of NRT and e-cigarettes would be associated with a $0.5 \%$ change in overall cigarette consumption. 


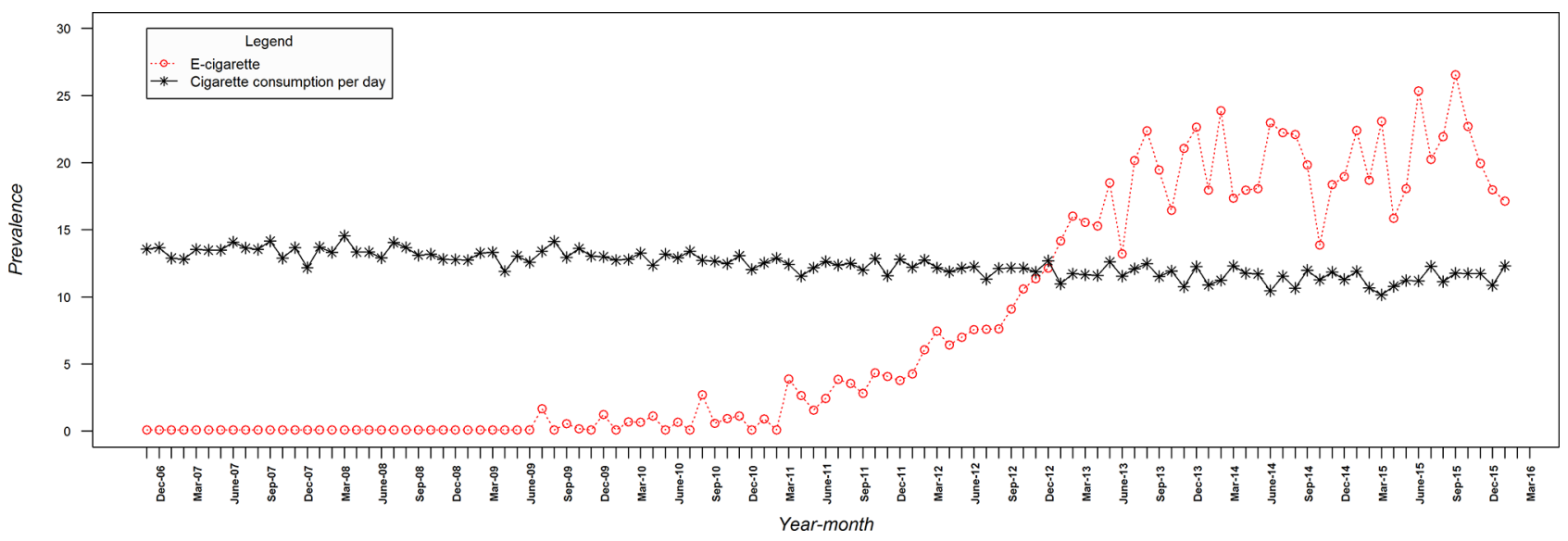

Figure 1 Monthly prevalence of cigarette consumption and e-cigarettes for harm reduction among smokers.

Strengthening the Reporting of Observational Studies in Epidemiology guidelines for the reporting of observational studies were followed throughout. ${ }^{36}$

\section{Sample size}

Simulation-based power analyses suggested that this study would have $80 \%$ power to detect a change in the output series of $0.009 \%$ for every $1 \%$ change in the input series, assuming 113 monthly data collection points, MA (1) autocorrelation, ${ }^{37}$ a baseline proportion for the input series of $0.005,{ }^{9}$ a baseline mean (SD) for the output series of $12.3^{38}$ and a total change over time for the input series of $30 \%$. $^{38}$

\section{RESULTS}

\section{Sample characteristics}

Data were collected on 199483 adults aged 16+ years taking part in the STS who reported their smoking status between November 2006 and March 2016. Of these, 43608 (20.8\%, 95\% CI 20.6 to 21.0$)$ were current smokers. Fifty-two per cent (95\% CI $52 \%$ to $53 \%$ ) of the smokers were male and $60.4 \%$ (95\% CI $60 \%$ to $60.1 \%$ ) were in routine or manual positions or were unemployed.
The average age of smokers in this study was 42.1 years (95\% CI 42.0 to 42.1 ).

\section{Main analysis}

Figure 1 shows that cigarette consumption declined over the study period from 13.6 to 12.3 (mean 12.4, SD 0.92). This figure also shows that current use of e-cigarettes among smokers for harm reduction increased from negligible use in the last quarter of 2006 to $17.1 \%$ at the end of the study (mean 7.8\%, SD 8.82). Figure 2 shows that there was also a decline in the use of NRT for harm reduction from $12.2 \%$ to $6 \%$ (mean $14.4 \%$, SD 4.36). Online supplementary figures 1 and 2 show the changes in e-cigarette and NRT use for smoking reduction and temporary abstinence, respectively.

Tables 1, 2 and 3 show the results of the ARIMAX models assessing the association between cigarette consumption per day with (1) e-cigarette use among current smokers and NRT use for harm reduction; (2) e-cigarette and NRT use for smoking reduction and (3) e-cigarette and NRT use for temporary abstinence. The findings were inconclusive as to whether an association was present between use of e-cigarettes and NRT for any purpose and cigarette consumption.

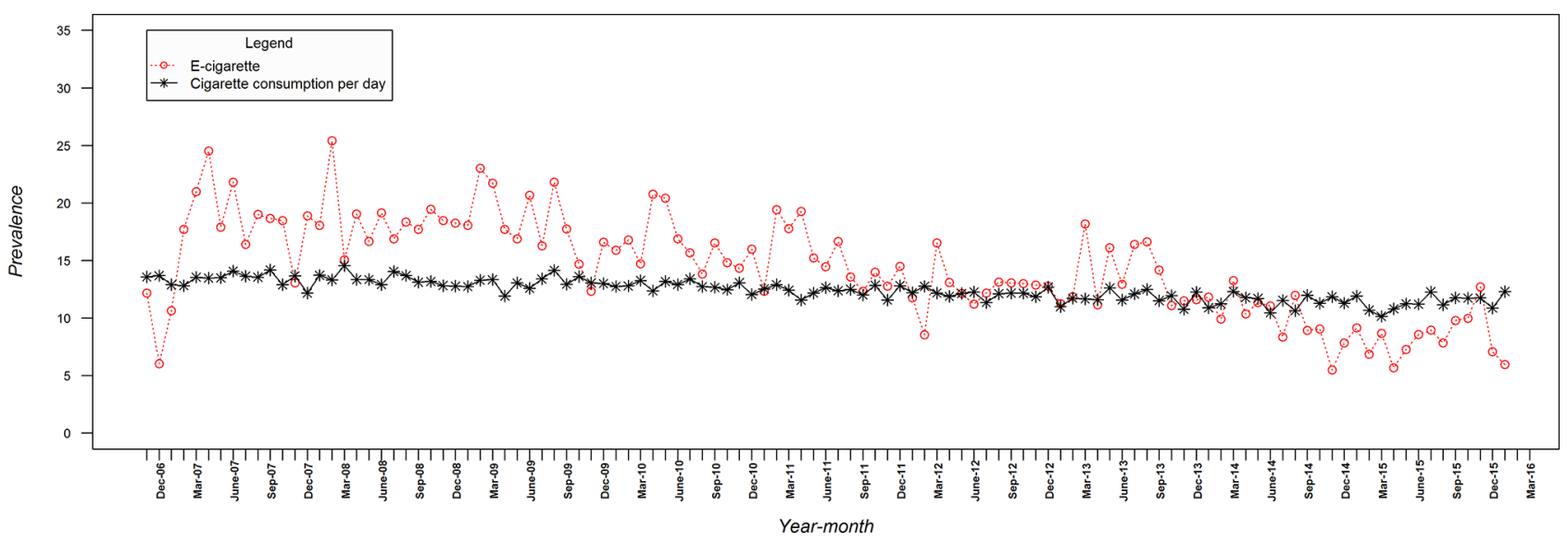

Figure 2 Monthly prevalence of cigarette consumption and nicotine replacement therapy use for harm reduction among smokers. 


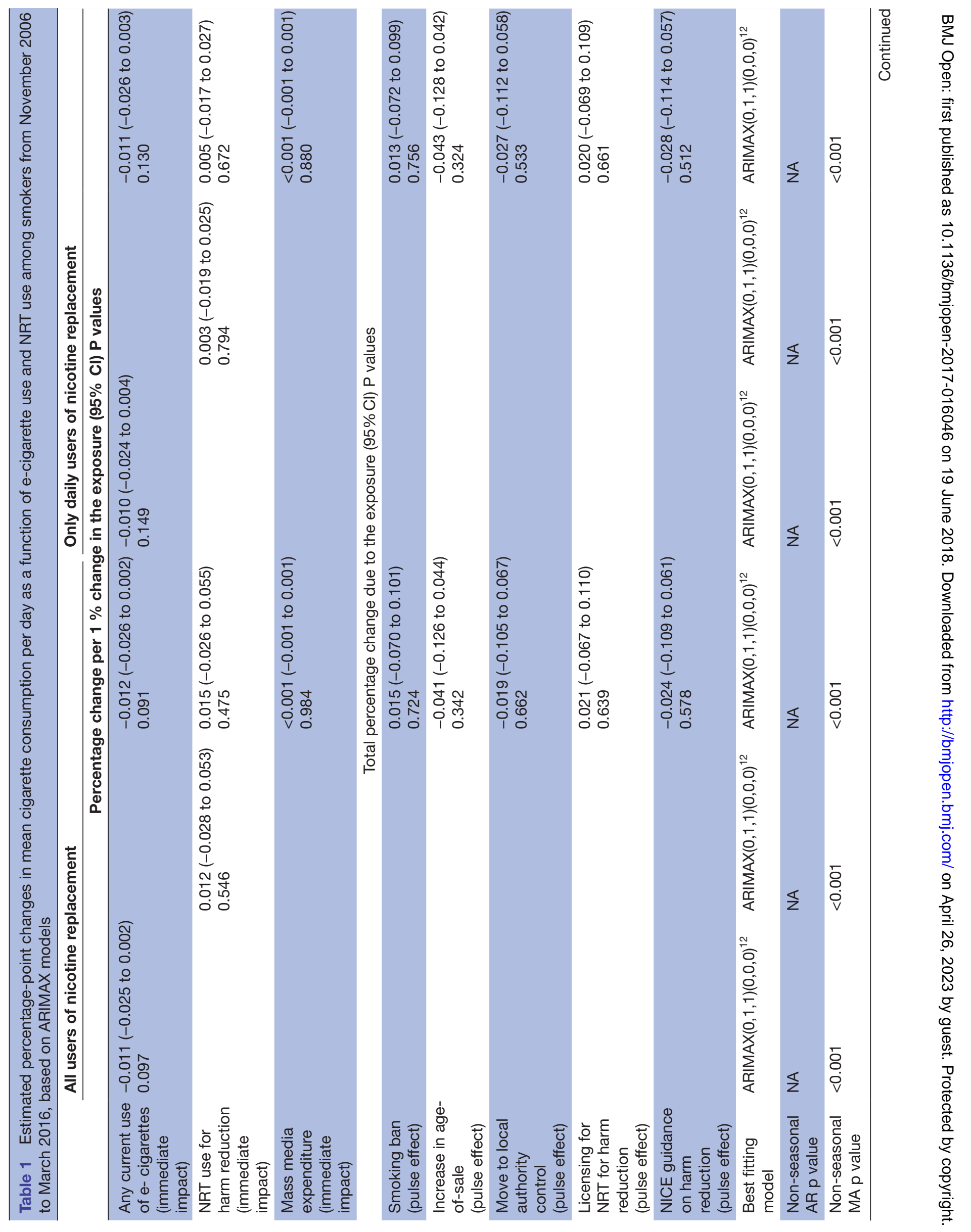




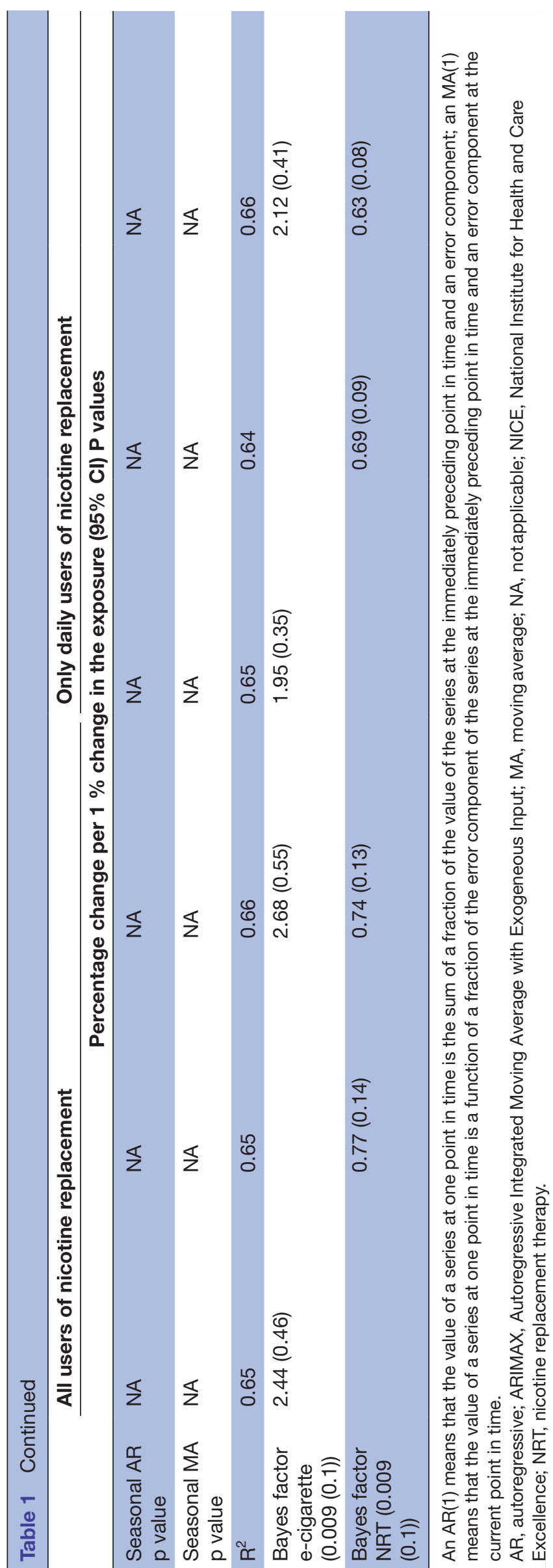

BFs were between one-third and three when assuming a $0.009 \%$ change in cigarette consumption for every percentage change in the input series, suggesting the data are insensitive to detect very small reductions in cigarette consumption. Most BFs were less than one-third, when assuming a $0.1 \%$ change in cigarette consumption for every percentage change in the input series, suggesting evidence for the null hypothesis that NRT use and e-cigarette use among smokers has not resulted in large reductions in cigarette intake.

\section{Sensitivity analysis}

Current daily use of e-cigarettes among smokers for harm reduction increased from negligible use in the last quarter of 2006 to $11.1 \%$ at the end of the study (mean $4.5 \%, \mathrm{SD} 4.91)$. There was also an increase in e-cigarette use specifically for temporary abstinence (from $0.1 \%$ to 8.4\%; mean 3.5\% SD 3.81) and smoking reduction (from $0.1 \%$ to $8.3 \%$; mean $3.3 \%$ SD 3.64 ).

In contrast, there was a decline in the use of NRT for harm reduction from $7.3 \%$ to $2.9 \%$ (mean $6.5 \%$, SD 2.35) and a decline in NRT use specifically for temporary abstinence (from $7.3 \%$ to $1.8 \%$; mean $4.7 \%$ SD 2.29) and smoking reduction (from $6.8 \%$ to $2.6 \%$; mean $5.8 \%$, SD 2.46).

Tables 1, 2 and 3 also show the results of the sensitivity analyses restricted to those smokers using NRT or e-cigarettes daily. The findings were inconclusive as to whether or not an association was present between the daily use of e-cigarettes and NRT for any purpose and cigarette consumption. BFs suggested the data are insensitive to detect very small reductions in cigarette consumption, but there is evidence for the null hypothesis that NRT use and e-cigarette use among smokers have not resulted in large reductions in cigarette intake.

\section{DISCUSSION}

To our knowledge, this is the first empirical study to estimate the population association between the use of e-cigarettes and NRT among current smokers on cigarette consumption per day, using a time-series approach. There was evidence that there was no substantial association between the rise in use of e-cigarettes and decline in NRT use and changes in cigarette consumption per day.

\section{Strengths and limitations}

A strength of the study is the use of a large representative sample of the population in England, stratification of results by daily use, and the consideration of both temporary abstinence and smoking reduction. Previous studies have shown that reductions in cigarette intake are dependent on the extent of NRT use and differ as a function of the specific harm-reduction behaviour, that is, an attempt to cut down or restraining from smoking during periods of brief abstinence. ${ }^{26}$

The study had a number of limitations. First, caution should be taken when interpreting estimates of the 


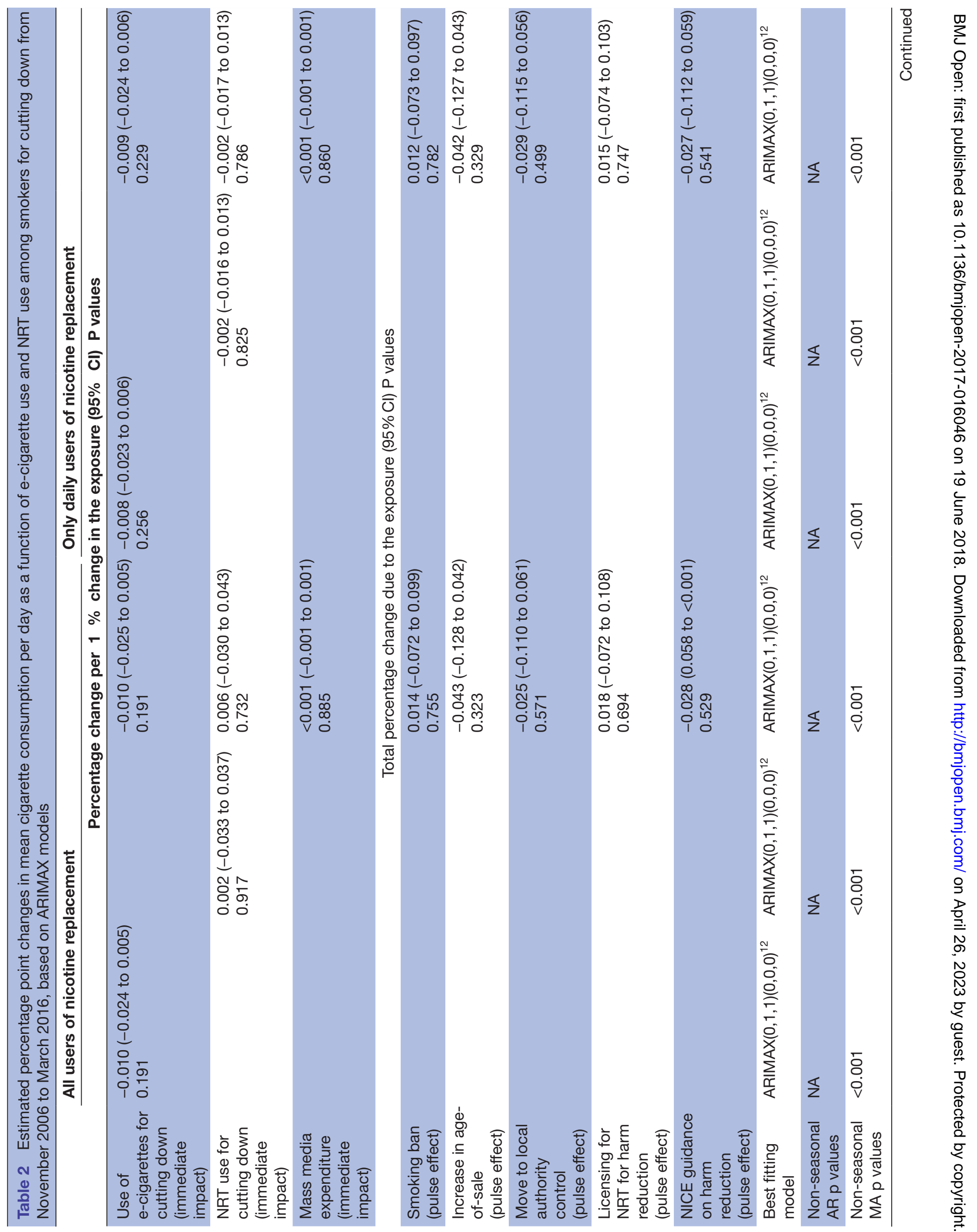


covariates, that is, impact of some of the tobacco-control policies, as interrupted explanatory variables with short time-periods prior to their introduction in ARIMAX-type models often give inaccurate estimates of the SEs. ${ }^{28}$ Thus, although the increase in age-of-sale has been previously associated with a decline in smoking prevalence, ${ }^{24}$ the short lead-in period may have masked any true association. ${ }^{27}$ Second, the STS required participants to recall their average daily cigarette intake which is likely to have been somewhat inaccurate. Third, the findings may not generalise to other countries. England has a strong tobacco-control climate and relatively liberal attitude towards harm reduction and e-cigarette use. In countries with weaker tobacco control, or stricter regulation of using products for harm reduction, different effects may be observed. Fourth, although we are unaware of any other major population-level interventions or other events during the study period, we cannot rule out residual confounding. Fifth, participants were not asked questions regarding potentially important features of the e-cigarette (eg, nicotine content, flavouring, device type) or frequency and duration of use. It is likely that these factors may play a role in their effectiveness and should be considered in future studies. ${ }^{1599}$ Finally, as data were not collected on current e-cigarette use prior to April 2011, prevalence was estimated from use during a quit attempt or from previous studies. ${ }^{21}{ }^{22}$ This was necessary to ensure that the time series was long enough for an ARIMAX analysis and is an appropriate approach when data are missing completely at random. ${ }^{16}{ }^{40}$ As prevalence was low and relatively stable during this period, it is unlikely to have impacted on the reported results.

\section{Implications of findings}

The findings are in line with previous studies which show that reductions in cigarette consumption observed in clinical trials of NRT for harm reduction do not appear to generalise beyond the closely controlled trial setting. ${ }^{12}$ It was hypothesised that e-cigarettes may be associated with population mean cigarette intake given that they reduce cravings more effectively than NRT, ${ }^{7}{ }^{10} 11$ have better adherence rates ${ }^{712}$ and deliver clinically significant levels of nicotine into the blood. ${ }^{1011113}$

The finding that e-cigarette use was not associated with reductions in consumption at a population level is consistent with previous real-world studies at the individual level. These have found little change in consumption among ever e-cigarette users ${ }^{41}$ and that only a minority of daily users manage to reduce by a substantial amount which is not likely to be detected at a population level. ${ }^{42}$ The findings of a recent pragmatic controlled trial, whereby $60 \%$ of participants using e-cigarettes had managed to reduce by over $50 \%$ by 6 months' follow-up, suggests that the lack of effectiveness at a population level may not be the consequence of poor behavioural support. ${ }^{11}$

Of course, it remains plausible that e-cigarettes may still be associated with a small effect on mean population cigarette consumption, ${ }^{15}$ and that a reduction in harm from 


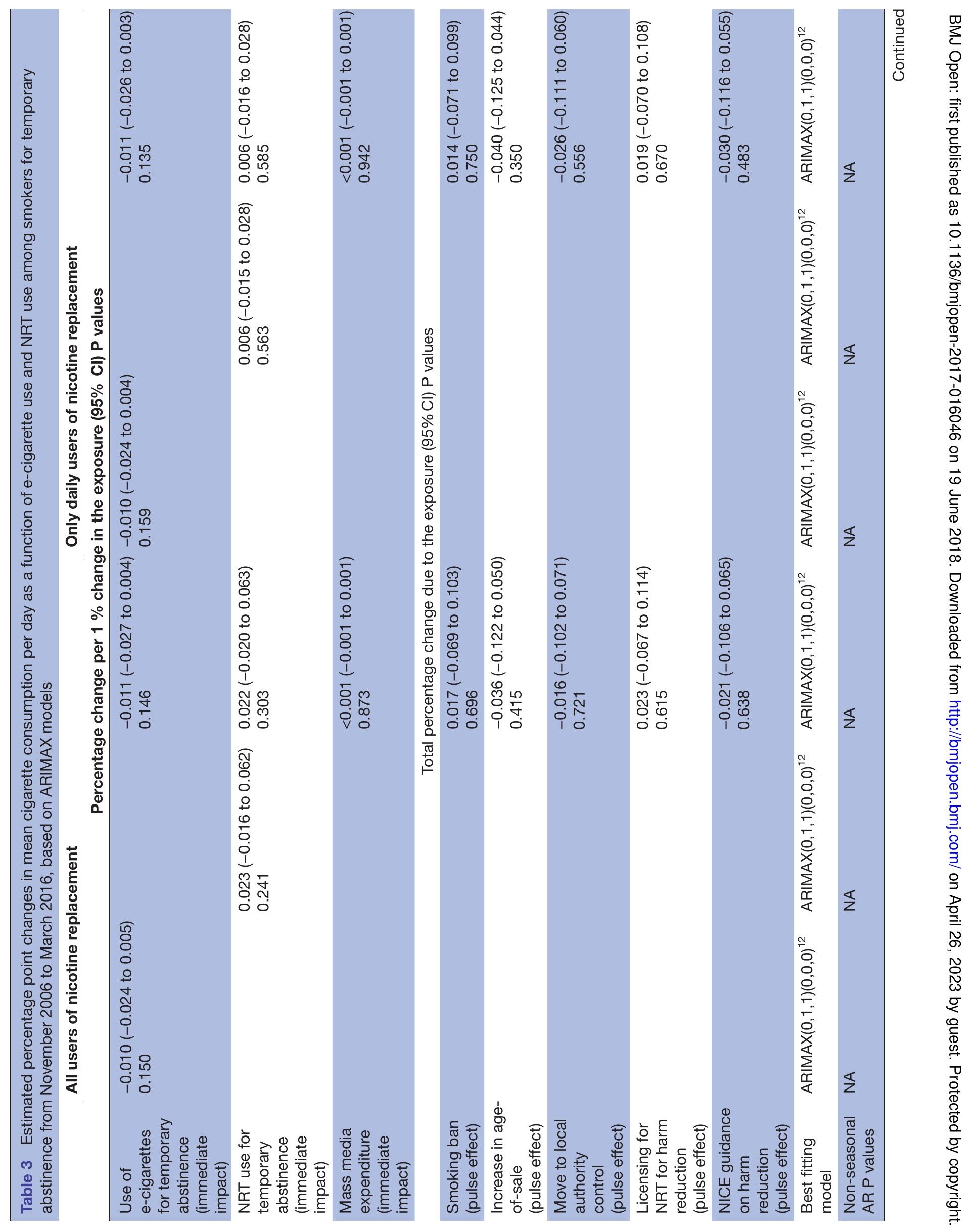



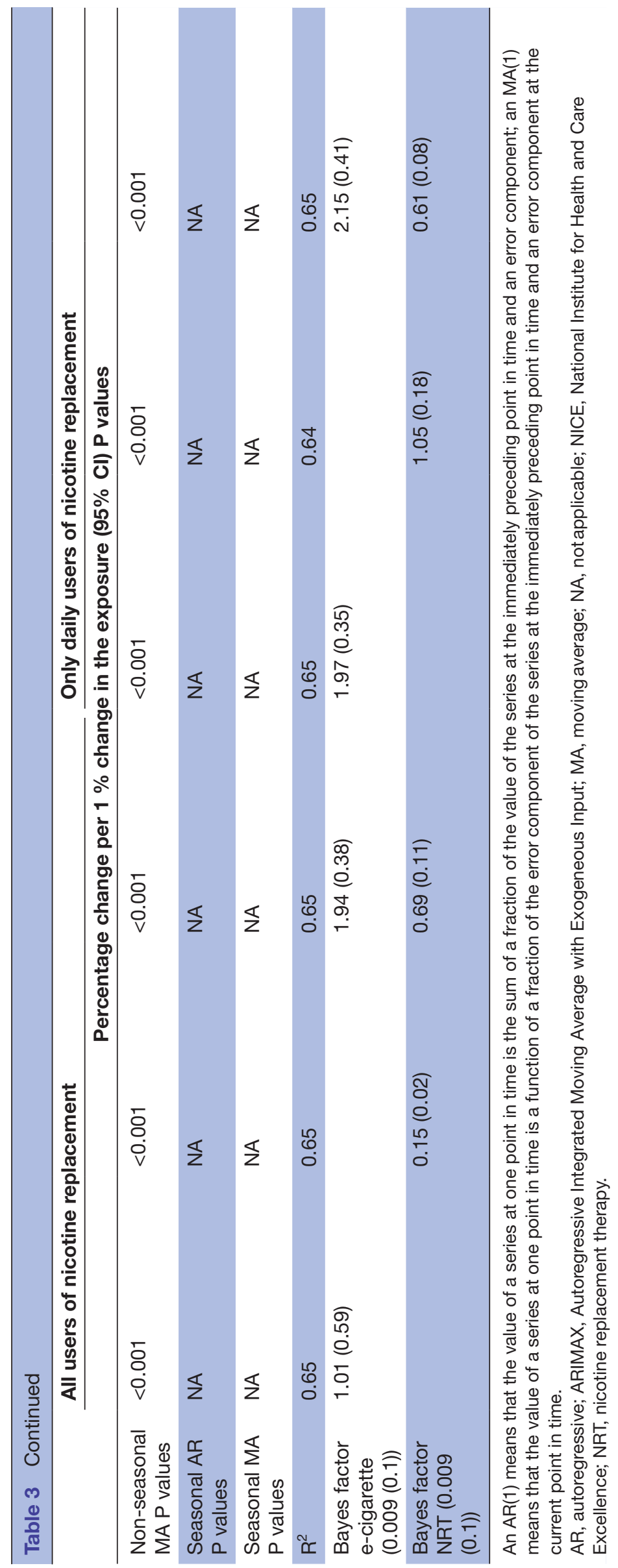

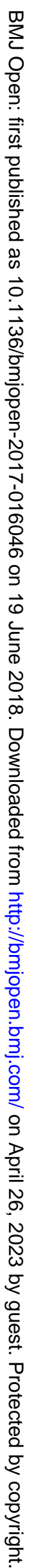


smoking at a population level could be seen through their promotion of quit attempts ${ }^{37}$ or by reducing smoke intake from each cigarette. ${ }^{5}$

\section{Conclusion}

In conclusion, the increased prevalence of e-cigarettes use among smokers in England has not been associated with a detectable change in cigarette consumption per day. The decline in the use of NRT has also not been associated with a change in mean cigarette intake. If use of e-cigarettes and licensed NRT while smoking act to reduce cigarette consumption, the effect is probably small.

Contributors EB, JB, SM and RW designed the study. EB wrote the first draft and conducted the analyses. All authors commented on this draft and contributed to the final version.

Funding The Smoking Toolkit Study is currently primarily funded by Cancer Research UK (C1417/A14135; C36048/A11654; C44576/A19501) and has previously also been funded by Pfizer, GSK and the Department of Health. JB's post is funded by a fellowship from the Society for the Study of Addiction and CRUK also provides support (C1417/A14135). RW is funded by Cancer Research UK (C1417/A14135). EB is funded by a fellowship from the NIHR SPHR (SPHRSWP-ALC-WP5) and CRUK also provides support (C1417/A14135). SW is funded by Cancer Research UK (C1417/A14135) and NIHR SPHR (SPHR-SWP-ALC-WP5) also provide support. SPHR is a partnership between the Universities of Sheffield; Bristol; Cambridge; Exeter; UCL; The London School for Hygiene and Tropical Medicine; the LiLaC collaboration between the Universities of Liverpool and Lancaster and Fuse; The Centre for Translational Research in Public Health, a collaboration between Newcastle, Durham, Northumbria, Sunderland and Teesside Universities.

Disclaimer The views expressed are those of the authors(s) and not necessarily those of the NHS, NIHR or Department of Health. No funders had any involvement in the design of the study, the analysis or interpretation of the data, the writing of the report or the decision to submit the paper for publication.

Competing interests RW undertakes consultancy and research for and receives travel funds and hospitality from manufacturers of smoking cessation medications but does not, and will not take funds from e-cigarettes manufacturers or the tobacco industry. RW and SM are honorary co-directors of the National Centre for Smoking Cessation and Training. RW is a Trustee of the stop-smoking charity, QUIT. RW's salary is funded by Cancer Research UK. SM's salary is funded by Cancer Research UK and by the National Institute for Health Research (NIHR)'s School for Public Health Research (SPHR). EB and JB have received unrestricted research funding from Pfizer. EB and JB are funded by CRUK. EB is also funded by NIHR's SPHR and JB by the Society for the Study of Addiction. RW has received travel funds and hospitality from, and undertaken research and consultancy for pharmaceutical companies that manufacture or research products aimed at helping smokers to stop. These products include nicotine replacement therapies, Champix (varenicline) and Zyban (bupropion). This has led to payments to him personally and to his institution.

Patient consent Obtained.

Ethics approval Ethical approval for the Smoking Toolkit Study was granted by the UCL Ethics Committee (ID 0498/001).

Provenance and peer review Not commissioned; externally peer reviewed.

Data sharing statement For access to the data please contact the lead author, EB (e.beard@ucl.ac.uk).

Open access This is an open access article distributed in accordance with the terms of the Creative Commons Attribution (CC BY 4.0) license, which permits others to distribute, remix, adapt and build upon this work, for commercial use, provided the original work is properly cited. See: http://creativecommons.org/ licenses/by/4.0/

(c) Article author(s) (or their employer(s) unless otherwise stated in the text of the article) 2018. All rights reserved. No commercial use is permitted unless otherwise expressly granted.

\section{REFERENCES}

1. Moore D, Aveyard P, Connock M, et al. Effectiveness and safety of nicotine replacement therapy assisted reduction to stop smoking: systematic review and meta-analysis. BMJ 2009;338:b1024.

2. Beard E, McNeill A, Aveyard P, et al. Use of nicotine replacement therapy for smoking reduction and during enforced temporary abstinence: a national survey of English smokers. Addiction 2011;106:197-204.

3. Beard E, Michie S, Fidler J, et al. Use of nicotine replacement therapy in situations involving temporary abstinence from smoking: a national survey of English smokers. Addict Behav 2013;38:1876-9.

4. Beard E, Aveyard P, Michie S, et al. Does use of nicotine replacement therapy while continuing to smoke undermine cessation?: a systematic review. J Smok Cessat 2013;8:45-56.

5. Beard E, Vangeli E, Michie S, et al. The use of nicotine replacement therapy for smoking reduction and temporary abstinence: an interview study. Nicotine Tob Res 2012;14:849-56.

6. Beard E, Bruguera C, McNeill A, et al. Association of amount and duration of NRT use in smokers with cigarette consumption and motivation to stop smoking: a national survey of smokers in England. Addict Behav 2015;40:33-8.

7. Etter JF, Bullen C. Electronic cigarette: users profile, utilization, satisfaction and perceived efficacy. Addiction 2011;106:2017-28.

8. Etter J-F. Electronic cigarettes: a survey of users. BMC Public Health 2010;10:1.

9. Beard E, Brown J, McNeill A, et al. Has growth in electronic cigarette use by smokers been responsible for the decline in use of licensed nicotine products? Findings from repeated cross-sectional surveys. Thorax 2015;70:974-8.

10. Vansickel AR, Cobb CO, Weaver MF, et al. A clinical laboratory model for evaluating the acute effects of electronic "cigarettes": nicotine delivery profile and cardiovascular and subjective effects. Cancer Epidemiol Biomarkers Prev 2010;19:1945-53.

11. Bullen $\mathrm{C}$, Howe $\mathrm{C}$, Laugesen $\mathrm{M}$, et al. Electronic cigarettes for smoking cessation: a randomised controlled trial. The Lancet 2013;382:1629-37.

12. Kralikova E, Kubatova S, Truneckova K, et al. The electronic cigarette: what proportion of smokers have tried it and how many use it regularly? Addiction 2012;107:1528-9.

13. Dawkins L. Electronic cigarettes: what are they and are they effective? E-Cigarette Summit, London, UK: (oral presentation). 2013 http://e-cigarette-summit.com/wp-content/uploads/2013/12/ Summit-Presentations.pdf

14. Polosa R, Caponnetto P, Morjaria JB, et al. Effect of an electronic nicotine delivery device (e-Cigarette) on smoking reduction and cessation: a prospective 6-month pilot study. BMC Public Health 2011;11:1.

15. McRobbie H, Bullen C, Hartmann-Boyce J, et al. Electronic cigarettes for smoking cessation and reduction. Cochrane Database Syst Rev 2014.

16. Box GE, Jenkins GM, Reinsel GC. Time series analysis: forecasting and control: John Wiley \& Sons, 2011.

17. Gallus $S$, Schiaffino A, La Vecchia C, et al. Price and cigarette consumption in Europe. Tob Control 2006;15:114-9.

18. Langley T, Szatkowski L, Lewis S, et al. The freeze on mass media campaigns in England: a natural experiment of the impact of tobacco control campaigns on quitting behaviour. Addiction 2014;109:995-1002.

19. Langley TE, Huang Y, McNeill A, et al. Prescribing of smoking cessation medication in England since the introduction of varenicline. Addiction 2011;106:1319-24.

20. Fidler JA, Shahab L, West O, et al. 'The smoking toolkit study': a national study of smoking and smoking cessation in England. BMC Public Health 2011;11:479.

21. Regan AK, Promoff G, Dube SR, et al. Electronic nicotine delivery systems: adult use and awareness of the 'e-cigarette' in the USA. Tob Control 2013;22:19-23.

22. Cho JH, Shin E, Moon SS. Electronic-cigarette smoking experience among adolescents. J Adolesc Health 2011;49:542-6.

23. Wakefield MA, Durkin S, Spittal MJ, et al. Impact of tobacco control policies and mass media campaigns on monthly adult smoking prevalence. Am J Public Health 2008;98:1443-50.

24. Health and Social Care Information Centre. NHS stop smoking services collection. 2015 www.hscic.gov.uk/stopsmoking

25. Hackshaw L, McEwen A, West R, et al. Quit attempts in response to smoke-free legislation in England. Tob Control 2010;19:160-4.

26. Beard E, Bruguera C, Brown J, et al. Was the expansion of the marketing license for nicotine replacement therapy in the United kingdom to include smoking reduction associated with changes in use and incidence of quit attempts? Nicotine Tob Res 2013;15:1777-81. 
27. NICE. NICE guidelines [PH45]: Smoking: Harm reduction. 2013 https://www.nice.org.uk/guidance/ph45.

28. Fidler JA, West R. Changes in smoking prevalence in 16-17-year-old versus older adults following a rise in legal age of sale: findings from an English population study. Addiction 2010;105:1984-8.

29. R Development Core Team. A language and environment for statistical computing. R foundation for statistical computing, Vienna, Austria. 2008 http://www.R-project.org

30. Wakefield MA, Coomber K, Durkin SJ, et al. Time series analysis of the impact of tobacco control policies on smoking prevalence among Australian adults, 2001-2011. Bull World Health Organ 2014;92:413-22.

31. Cryer JD, Chan K-S. Time series analysis - with applications in R. London: Springer-Verlag New York, 2008.

32. Yalta AT. Analyzing energy consumption and GDP nexus using maximum entropy bootstrap: the case of Turkey. Energy Economics 2011;33:453-60.

33. Granger CWJ. Some recent development in a concept of causality. $J$ Econom 1988;39:199-211.

34. Box GEP, Tiao GC. Intervention analysis with applications to economic and environmental problems. J Am Stat Assoc 1975;70:70-9.

35. Dienes Z. Using Bayes to get the most out of non-significant results. Front Psychol 2014;5.

36. von Elm E, Altman DG, Egger M, et al. The Strengthening the Reporting of Observational Studies in Epidemiology (STROBE) statement: guidelines for reporting observational studies. Prev Med 2007;45:247-51.

37. Beard E, West R, Michie S, et al. Association between electronic cigarette use and changes in quit attempts, success of quit attempts, use of smoking cessation pharmacotherapy, and use of stop smoking services in England: time series analysis of population trends. BMJ 2016;354:i4645.

38. Kuipers MA, Beard E, Hitchman SC, et al. Impact on smoking of England's 2012 partial tobacco point of sale display ban: a repeated cross-sectional national study. Tob Control 2017;26.

39. Hitchman SC, Brose LS, Brown J, et al. Associations between e-cigarette type, frequency of use, and quitting smoking: findings from a longitudinal online panel survey in great Britain. Nicotine Tob Res 2015;17:1187-94.

40. Little RJ, Rubin DB. Statistical analysis with missing data: John Wiley \& Sons, 2014.

41. Shi Y, Pierce JP, White M, et al. E-cigarette use and smoking reduction or cessation in the 2010/2011 TUS-CPS longitudinal cohort. BMC Public Health 2016;16:1105.

42. Brose LS, Hitchman SC, Brown J, et al. Is the use of electronic cigarettes while smoking associated with smoking cessation attempts, cessation and reduced cigarette consumption? A survey with a 1-year follow-up. Addiction 2015;110:1160-8. 